\title{
Le Thesaurus de l'Image Médicale de Strasbourg
}

\section{Le TIMS pourquoi ?}

C'est grâce aux rédacteurs en chef de "Orthopédie - Traumatologie", $\mathrm{P}$ Kehr et $\mathrm{H}$ Coudane, que nous avons l'occasion de rapporter régulièrement des images provenant du TIMS (Thesaurus de l'Image Médicale de Strasbourg). Quatre grands répertoires ou index peuvent être consultés :

\section{Index alphabétique de mots clés :}

Si d'entrée de jeu je propose de créer un thésaurus d'images clés, cette création matérielle d'images doit avoir la contrepartie d'un très vaste index alphabétique de mots clés permettant la consultation directe d'images numérotées. Chaque mot clé conduit à un ou à plusieurs numéros d'images.

Exemple : Recklinghausen. Le mot clé de l'index conduit à la photographie de Recklinghausen, à une image clé de neurinome de l'acoustique, une dysplasie de l'orbite, etc.

\section{Répertoire numéroté :}

Le thesaurus contient un matériel théorique pouvant aller jusqu'à 300000 ou 500000 (?) images médicales. Le classement est très simple. Chaque image est classée linéairement au fur et à mesure de son arrivée. Les index et répertoires conduisent à l'image numérotée, qu'il s'agisse d'un catalogue d'images miniaturisées (déjà fonctionnel à l'heure actuelle) ou d'un stockage d'images informatisées sur vidéodisque ou disque numérique. Le même répertoire numéroté fait appel aux légendes ci-dessous (3):
A : 1 à 10000
$\mathrm{B}: 1$ à 10000
$\mathrm{C}: 1$ à 10000
etc ...

3. Répertoire numéroté des légendes:
$\mathrm{A}: 1$ à 10000
$\mathrm{B}: 1$ à 10000
$C: 1$ à 10000
etc ...

Les légendes sont courtes, traduites en plusieurs langues, anglais et allemand me paraissant être indispensables à côté du français et conduisent grâce au numéro à l'image et à sa légende trilingue.

4. Index alphabétique des disciplines, spécialités et activité : Les activités, spécialités et disciplines médicales sont présentées en fonction de la pratique. La classification est purement alphabétique et utilitaire.
Anatomie
Bactériologie
Cardiologie

Dans chaque activité nous prenons l'ordre alphabétique pour conduire au numéro de l'image.

\section{LE THESAURUS DE L'IMAGE MEDICALE DE STRASBOURG (TIMS)}

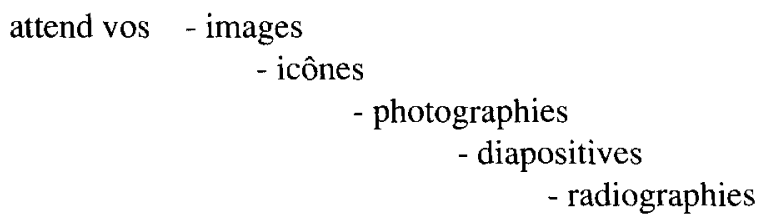

envoyez les avec légende à l'adresse suivante :

Professeur A. WACKENHEIM

Radiologie B

Pavillon Clovis Vincent

HUS de STRASBOURG

B.P. $n^{\circ} 426$

67091 STRASBOURG Cedex 